\title{
Effect of Dietary Supplementation with Short-Chain Fructooligosaccharides in Lactating Sows and Newly Weaned Piglets on Reproductive Performance of Sows, Immune Response and Growth Performance of Piglets from Birth to Slaughter
}

\author{
E Apper ${ }^{1}$, C Meymerit ${ }^{2}$, JC Bodin ${ }^{3}$, F Respondek ${ }^{1}$ and A Wagner ${ }^{1}$ \\ ${ }^{1}$ Tereos, Z.I. Portuaire, Marckolsheim, France \\ ${ }^{2}$ Interface Elevage, Route de Pau, Vignes, France \\ ${ }^{3}$ Jefo Europe, Rue Claude Chappe, Carquefou, France
}

Corresponding author: Apper E, Tereos, Z.I. portuaire, 67390 Marckolsheim, France, Tel: +33 388581611 ; Fax: +33 388586061 ; E-mail: Emmanuelle.apper-bossard@tereos.com

Received Date: July 28, 2016; Accepted Date: October 18, 2016; Published Date: October 25, 2016

Copyright: (C) 2016 Apper E, et al. This is an open-access article distributed under the terms of the Creative Commons Attribution License, which permits unrestricted use, distribution, and reproduction in any medium, provided the original author and source are credited.

Citation: Apper E, Meymerit C, Bodin JC, Respondek F, Wagner A. Effect of Dietary Supplementation with Short-Chain Fructooligosaccharides in Lactating Sows and Newly Weaned Piglets on Reproductive Performance of Sows, Immune Response, and Growth Performance of Piglets from Birth to Slaughter. J Anim Res Nutr 2016, 1: 18.

\section{Abstract}

Objective: The effects of an early-life dietary supplementation with short-chain fructooligosaccharides (scFOS) on immune response and growth performance of pigs from birth to slaughter were evaluated. The hypothesis was that scFOS have long-term effects on immune response, and growth performance.

Methods: One hundred and twenty-nine sows received a control diet (CTRL, $10 \mathrm{~g} / \mathrm{d}$ maltodextrins) or a diet supplemented with scFOS $(10 \mathrm{~g} / \mathrm{d})$ from d 109 of gestation to the weaning of the piglets.

After weaning, the piglets of each litter were divided into two groups to receive the CTRL or the scFOS diet $(1.2 \mathrm{~g} / \mathrm{d}$ maltodextrin or scFOS) during the pre-starter period. At 53 and $74 \mathrm{~d}$ of age, the piglets were vaccinated against Influenza.

Performance of sows and composition of colostrum were measured. Blood serum of the piglets was sampled one $d$ prior vaccination, at $d 74$ and 95 to measure Influenza-IgG levels. The growth parameters of the piglets were recorded from birth to slaughter.

Results: Supplementation of the sows with scFOS decreased farrowing duration $(p=0.012)$, colostrum fat content $(p=0.006)$ and increased IgG content in colostrum of sows with parity of 4 or higher $(p=0.006)$ without modifying the performance of suckling piglets. Back fat thickness at weaning tended to be higher in supplemented sows $(p=0.091)$. Diet with scFOS improved growth performance during the breeding period, resulting in reduced feeding time needed to reach a $120 \mathrm{~kg}$ body weight at slaughter. Piglets fed scFOS had higher Influenza-IgG titer $(p=0.05)$ after vaccination against Influenza virus.
Conclusion: These data suggest scFOS supplementation of sows during suckling period and of piglets during the postweaning period leads to long-term positive effects on growth of fattening pigs and to better physiological conditions of sows.

Keywords: Fructooligosaccharide; Immune response; Performance; Piglet; Prebiotic; Early life, Sow

\section{Abbreviations:}

BW: Body Weight; ADG: Average Daily Gain; ADI: Average Daily Intake; FCR: Feed Conversion Ratio; CTRL: Control; IgA and IgG: immunoglobulins $A$ and G; scFOS: Short-Chain Fructooligosaccharides

\section{Introduction}

Nutrition plays an essential role in developing microbiota and immune system of neonate piglets. Early-life nutrition may also influence gut immunity and metabolism at long-term and may impact growth of pigs at later physiological stages by imprinting [1]. Indeed, modulating the diet of females during gestation and lactation and/or the diet of young animal's results in later change in microbiota and epigenetic reactions in several species [2].

Nutrition of sows is crucial in preserving reproductive performance. Voluntary feed intake of highly prolific sows is generally insufficient to cover nutrient requirements for milk production and maintenance of body condition [3]. Furthermore, sows may become insulin-resistant during lactation [4] resulting in impairing their breeding career. Additionally, insulin-resistance of sows may decrease survival and growth performance of their suckling piglets. 
As prebiotics, short-chain fructooligosaccharides (scFOS) are "selectively fermented ingredients that induce specific changes in the composition or in the activity of the gastrointestinal microflora that confer benefits to the host" [5]. Supplementation with scFOS modulates gastrointestinal microbiota in pigs by selective stimulation of growth and activity of beneficial bacteria such as Bifidobacteria and Lactobacilli [6]. As a result, scFOS enhance integrity of the intestinal barrier of piglets [7] and increase insulin sensitivity in obese dogs [8]. Supplementation with scFOS of mothers and their young modulate immune response in puppies [9], while a supplementation of sows influences intestinal immune system maturation in piglets [10], thus suggesting that peri-partum supplementation with scFOS can modulate immune response. Furthermore, in the same study, a scFOS supplementation of sows modifies immune quality of colostrum and allows limiting loss of sow back fat at the end of the lactation, suggesting an improvement of the health status of the sow.

Due to the importance of early nutrition on later performance, and based on first encouraging results obtained by Le Bourgot et al. [10] on early gut maturation, a research program has been built to evaluate the effects of scFOS on later immune/metabolic response after a challenge and on later growth performance. In this context, Le Bourgot et al. [11] recently published data on effects of an early supplementation with scFOS on immune response after a vaccine against Influenza.

They demonstrated that post-weaning scFOS diet increased anti-influenza IgA levels in pig serum and faeces and that blood anti-influenza IgA levels were significantly and positively correlated to blood anti-Influenza IgG levels. In this study, sows were supplemented from the 4 last weeks of gestation to weaning at day 28 after farrowing; while piglets were supplemented until 77 days of age.

Due to the importance of early-life nutrition on later performance, the purpose of the present study was to perform similar trial with shorter supplementation duration for both sows and piglets and in field conditions.

The objective of the study was then to evaluate if supplementation with scFOS during early life of piglets would modulate their immune response and growth performance at longer term. Additionally, effects of scFOS supplementation on sow's reproductive performance were investigated.

\section{Materials and Methods}

The trial was conducted in France from September 2012 to May 2013, while it was only mandatory to obtain prior approval from ethical committee to conduct animal testing from January 2013.

However, the present experiment was conducted under supervision of veterinary Bernard Sansot, who is duly authorized to conduct animal testing on farm animals under the permission 5509 at Faculty of Medicine of Toulouse (France).

\section{Animals and experimental design}

The experiment was conducted in a commercial farm in France with 129 primiparous and multiparous sows (Large White $\times$ Landrace) in two replicates.

The sanitary condition of the farm and zootechnical performances were typical for France. A split-plot experimental design was used with sow diet effect in the main plots and differing piglet diet effect in the subplot.

Two diets were tested: a control diet (CTRL) delivered to 64 sows and the same basal diet supplemented with scFOS (scFOS) delivered to 65 sows. Five $d$ before parturition, sows were allocated to one of the two diets according to their parity.

Finally, 28 primiparous sows (13 CTRL and 15 FOS), 28 sows with parity 2 ( $n=14$ per diet), 24 sows with parity 3 ( $n=13$ CTRL and $n=11$ FOS), 26 sows with parity 4 ( $n=13$ per diet), 12 sows with parity 5 ( $n=6$ per diet) and 11 sows with parity 6 (5 CTRL and 6 FOS) were used.

The sows were in a group-housing room during gestation and moved to the farrowing room after $109 \pm 1 \mathrm{~d}$ of gestation to start the experiment. They were kept in individual farrowing crates (length: $2.7 \mathrm{~m}$; width: $1.7 \mathrm{~m}$ ).

For the first $48 \mathrm{~h}$ post-partum, the original litter was kept with the sow. Beyond $48 \mathrm{~h}$; litters were standardized to 12 piglets by cross-fostering within diet groups. Ambient temperature in the farrowing room was maintained between 22 and $25^{\circ} \mathrm{C}$.

Throughout the lactation period, piglets had no access to creep feed. They were weaned at the age of $20 \mathrm{~d}$. After weaning, half of the piglets were excluded from the experiment and left the farm. The other half remained in their original treatment group defined by their dam and was then subdivided into two groups to receive either the ScFOS diet or the CTRL diet from d21 to $d 40$.

The piglets stayed in a group housing room from post-weaning to slaughter. They received two injections of the vaccine Stellamune Mycoplasma, one during suckling period and the other after weaning.

No collective or systematic treatments were applied during the overall period. During the post-weaning phase, pigs were housed in pens that could house up to 32 piglets. In growing and finishing phases, the piglets were housed in pens that could house up to 16 piglets. Ambient temperature in the postweaning phase was maintained between 25 and $29^{\circ} \mathrm{C}$. The pigs were bred until a BW of $120 \mathrm{~kg}$ and then slaughtered in accordance with the current European regulations (EU regulations $1 / 2005$ and 1099/2009).

\section{Diets}

All diets were formulated according to nutrient and energy requirements of sows and piglets in respect of their physiological status (Tables 1 and 2). During gestation, all the sows received two daily meals at 7:30 AM and 2:00 PM. They were fed on average $2.5 \mathrm{~kg}$ (from d 1 to 36 ), $2.5 \mathrm{~kg}$ (from d 37 to 
80 ) and $3.5 \mathrm{~kg}$ (from d 81 to 109) daily of a conventional diet for pregnant sows.

From one week before parturition to weaning of piglets, sows were fed ad libitum a conventional diet for lactating sows (Table 1). This conventional diet was supplemented with either $10 \mathrm{~g} / \mathrm{d}$ maltodextrins (CTRL; Maldex 120, Tereos Syral, Marckolsheim, France; 13 Equivalent Dextrose on average, 1\% dextrose, 3\% maltose, $6 \%$ maltotriose and $90 \%$ higher sugars), which are digestible chains of glucose molecules linked by $\alpha-1-4$ bonds, or $10 \mathrm{~g} / \mathrm{d}$ scFOS (Profeed P95, Tereos Syral, Marckolsheim, France; $95 \%$ scFOS with molecular chain length from 3 to $5,37 \%$ GF2; $53 \%$ GF3 and $10 \%$ GF4), non-digestible chains of glucose and fructose linked by $ß(2-1)$ bonds.

Five diets were formulated for piglets: a pre-starter diet from weaning to $\mathrm{d} 40$, a starter diet from d 41 to 64; a pre-growing diet from d 65 to d 103; a growing diet from d 104 to 148, and a finishing diet until slaughter (Table 2). Fresh water was always freely available to the sows and piglets. From weaning to $d 40$ (i.e., for the pre-starter period), the piglets of each litter were divided into 2 groups.

They were fed with the conventional diet supplemented with either $1.2 \mathrm{~g} / \mathrm{d}$ maltodextrins or $1.2 \mathrm{~g} / \mathrm{d}$ scFOS. Maltodextrins and scFOS were directly incorporated in the diets. Finally, two groups of sows were formed according to their diets: CTRL and ScFOS groups.

Four groups of piglets were distinguished: a CTRL/CTRL group in which both the sows and their piglets received the control diet; a CTRL/scFOS group in which the sows were fed the control diet and their piglets received the scFOS diet; a scFOS/CTRL group in which the sows were fed the scFOS diet and their piglets received the control diet; a scFOS/scFOS group in which both the sows and their piglets received the scFOS diet.

Table 1: Chemical analysis of the experimental diets fed by sows during gestation and lactation.

\begin{tabular}{|c|c|c|}
\hline Item & Gestation diet & Lactation diet \\
\hline Corn & 44.5 & 40 \\
\hline Barley & 30 & \\
\hline Triticale & & 27.5 \\
\hline Sunflower meal & 12 & \\
\hline Soybean meal & 8.5 & 21.5 \\
\hline Tec Truie perfomance $5 \%{ }^{1}$ & 4.85 & 4.83 \\
\hline Tec Omega & & 2.5 \\
\hline Tec Lactation & & 2.5 \\
\hline Soya oil & & 1 \\
\hline Lys $\mathrm{HCl}$ & 0.15 & 0.15 \\
\hline Vitamin E50 & & 0.02 \\
\hline \multicolumn{3}{|l|}{ Chemical Analysis, \% as DM } \\
\hline $\mathrm{CP}$ & 15.9 & 19.8 \\
\hline Fat & 3 & 4.8 \\
\hline Crude fiber & 6.8 & 3.5 \\
\hline Ash & 6.5 & 8 \\
\hline $\mathrm{DM}, \%$ as fed & 87.3 & 87.4 \\
\hline $\mathrm{DE}^{2}, \mathrm{MJ} / \mathrm{kg}$ of $\mathrm{DM}$ & 15 & 16.4 \\
\hline Lys & 0.7 & 1.16 \\
\hline \multirow[t]{3}{*}{ Daily allowance, $\mathrm{kg}$} & d 1 to $d 36: 2.5$ & Ad libitum \\
\hline & d 37 to $d 80: 2.5$ & \\
\hline & d 81 to $d 112: 3.5$ & \\
\hline \multicolumn{3}{|c|}{ 1: Commercial products produced by Alitec, Vignes, France } \\
\hline \multicolumn{3}{|c|}{ 2: Digestible energy was estimated from INRA table } \\
\hline
\end{tabular}


Table 2: Composition of the experimental diets formulated for piglets and growing-finishing pigs.

\begin{tabular}{|c|c|c|c|c|c|}
\hline Period & Pre-starter & Starter & Pre-growing & Growing & Finishing \\
\hline Age of pigs, $d$ & $20-40$ & $41-64$ & $65-103$ & $104-148$ & 148-Slaughter \\
\hline Commercial product ${ }^{1}$ & 40 & 5 & 4 & 51 & 3 \\
\hline Triticale & 27 & 48.5 & 23 & & 31 \\
\hline Corn & 10 & 10 & 35 & 49 & 30 \\
\hline Barley & 10 & 10 & & & \\
\hline Soybean meal & 13 & 21.5 & 18 & & 12 \\
\hline Rapeseed meal & & 5 & 10 & & 14 \\
\hline $\mathrm{DM}, \%$ as fed & 88.7 & 86.9 & 87 & 87.2 & 86.9 \\
\hline \multicolumn{6}{|c|}{ Chemical Analysis, \% as DM } \\
\hline $\mathrm{CP}$ & 21 & 21.4 & 20.9 & 19.8 & 18.8 \\
\hline Fat & 7.4 & 2 & 2.7 & 3 & 3.6 \\
\hline Crude fiber & 4 & 4.2 & 4.5 & 5.6 & 4.4 \\
\hline Ash & 8 & 7.5 & 6.5 & 6.1 & 5.9 \\
\hline $\mathrm{DE}^{2}, \mathrm{MJ} / \mathrm{kg}$ of $\mathrm{DM}$ & 16.7 & 15.2 & 15.3 & 15.3 & 15.6 \\
\hline $\mathrm{NE}^{2}, \mathrm{MJ} / \mathrm{kg}$ of $\mathrm{DM}$ & 11.8 & 10.7 & 10.8 & 10.9 & 11.2 \\
\hline Lys & 1.59 & 1.4 & 1.2 & 1.11 & 0.94 \\
\hline Daily allowance, $\mathrm{kg}$ & 0.38 & 0.92 & 1.75 & 2.6 & 2.45 \\
\hline \multicolumn{6}{|c|}{$\begin{array}{l}\text { 1: Commercial products are produced by Alitec, Vignes, France and are Babytec for pre-starter, Porcitec wheat } 5 \% \text { for starter, Tec gras and Nourritec concentrate for pre- } \\
\text { growing, specific complementary feedstuff for growing and Tec gras for finishing. Babytec contained organic acids, and vegetal extracts. All these products contained a } \\
\text { premix with minerals and vitamins }\end{array}$} \\
\hline \multicolumn{6}{|c|}{ 2: Digestible energy (DE) and Net energy (NE) were estimated from INRA table } \\
\hline
\end{tabular}

\section{Vaccination challenge}

During the replication 2, 43 piglets (10 piglets for the CTRL/ scFOS group, 11 piglets in all other groups) were chosen according to their $\mathrm{BW}$ in order to receive vaccination against Influenza (Gripovac, Merial, France). Each 10/11 piglets of each diet were in the same pen and did not belong to the same litter. They received an intramuscular dose of vaccine at the age of 53 and $74 \mathrm{~d}$.

\section{Samples and measures}

Sows: The overall feed intake of the sows was recorded. Backfat thickness of each sow was ultrasonically measured (RENCO leanmeater, Minneapolis, USA) at farrowing and weaning of the piglets. Farrowing was assisted and the duration was registered. Some $30 \mathrm{~mL}$ colostrum samples from 16 and 17 sows for CTRL and ScFOS diets respectively were collected $1 \mathrm{~h}$ after the birth of the last piglet. Colostrum was extracted from the 4 teats located in the middle parts of the udder [12]. Colostrum samples were immediately frozen at $-20^{\circ} \mathrm{C}$ until further analysis.

Piglets: The number of piglets and the mortality rate were recorded at birth, at $48 \mathrm{~h}$, and at the age of $21 \mathrm{~d}$. Average Body Weight (BW) of each litter was measured at birth, and at weaning. BW, average daily intake (ADI), average daily gain (ADG), feed conversion ratio (FCR) and mortality were recorded per pen on day 40,64, 103 and 148 . The pigs were slaughtered when they reached a body weight of $120 \mathrm{~kg}$. Ages at slaughter, lean meat content and carcass weight were individually recorded. The percentages of fat and muscle per carcass were also individually determined by the optical sensor (Sydel, Lorient, France) used in the slaughter house. During vaccination challenge, $3 \mathrm{~mL}$ of blood from the 43 vaccinated piglets were sampled from the jugular vein one $d$ before the first injection, at the age of 52 (prior the vaccination), 74 and $95 \mathrm{~d}$, using a syringe without conservator nor anticoagulant (Terumo Neosafe, Guyancourt, France). Blood was centrifuged and resulting serum was stored at $-20^{\circ} \mathrm{C}$ for later determination of Influenza-specific IgG.

\section{Chemical analysis of colostrum}

Fat content was measured according to the SchmidBondzynski-Ratzlaff method (NF V04-286). Crude Protein (CP) content was determined according to the Kjeldahl method (NF V04-407). Total carbohydrates were deduced by calculation based on difference with other nutrient contents (Laboratoire de Développement et d'Analyses des Côtes d'Armor (LDA, Ploufragran, France)). Dry Matter (DM) was obtained after $103^{\circ} \mathrm{C}$ heating for $6 \mathrm{~h}$. The samples were weighed before and after heating. DM was deduced by difference of weights. Ash content was obtained by calcination at $550^{\circ} \mathrm{C}$ for $4 \mathrm{~h}$. The samples were weighed before and after calcination. Ash content was deduced 
from the difference in weights. Energy value was calculated according to the following equation: energy expressed as $\mathrm{kJ} / 100$ $\mathrm{g}=9 \times$ Fat content $+4 \times \mathrm{CP}+4 \times$ Total carbohydrates

\section{Immunological analysis}

The levels of Immunoglobulin A (IgA) and IgG in colostrum were quantitated by ELISA according to immunoglobulin standards from the Laboratoire de Développement et d'Analyses des Côtes d'Armor (LDA, Ploufragran, France).

Blood levels of Influenza-specific IgG were determined by indirect hemagglutination test according to the standard procedure [13] adapted by the Laboratoire de Développement et d'Analyses des Côtes d'Armor (LDA, Ploufragran, France).

\section{Statistical analysis}

The observations on the sows, including feed intake, back fat thickness, litter characteristics at birth and at weaning, colostrum composition, and farrowing duration were analyzed by ANOVA using the GLM procedure [14] with sow as the experimental unit. The model included the diet effects, parity and replicate, and the interaction between diet and parity and between diet and replicate. To determine colostrum composition, litter size effect was firstly included but was not significant and removed from the model.

For all the parameters measured on all sows, except for colostrum data, 5 classes of parity were formed: class 1: parity 1 , $n=14$ sows in CTRL group and 15 sows in scFOS group; class 2: parity $2, n=14$ sows per dietary treatment; class 3 : parity $3, n=10$ sows in CTRL group and 13 sows in ScFOS group; class 4: parity 4, $\mathrm{n}=13$ sows per dietary treatment; class 5 : parity 5 and more, $\mathrm{n}=$ 11 sows in CTRL group and 12 sows in ScFOS group).

According to the distribution of the 33 values obtained to evaluate colostrum composition, four classes of parity were identified: class 1 : parity $1, n=4$ sows per dietary treatment; class 2: parity $2, \mathrm{n}=4$ sows per dietary treatment; class 3 : parity $3, n=4$ sows per dietary treatment; class 4 : parity 4 and more, $\mathrm{n}=4$ sows in CTRL group and 5 sows in scFOS group.

Data on growth performance on $d 40,64,103$ ( $n=28$ pens) and 148 ( $n=56$ pens) were analyzed by ANOVA using the GLM procedure [14] with pen as the experimental unit in a completely randomized design. The residual error was used to test the main effect of dietary treatments. The model included the effects of the diet of the sows, the diet of the piglets, replicate, piglet sex and the interactions between diet of sows and diet of piglets, diet of sow and piglet sex, and diet and sex of piglet.
Data on fattening pigs at slaughtering were analyzed by ANOVA using the GLM procedure [14] with pig as the experimental unit in a completely randomized design. The residual error was used to test the main effect of dietary treatments. The model included the effects of the diet of the sows, the diet of the piglets, replicate, piglet sex and the interactions between diet of sows and diet of piglets, diet of sow and piglet sex, and diet and sex of piglet. Means were compared using orthogonal polynomials [15]. When an interaction was significant, this was specified in the text. Data were presented as means \pm Standard Deviation (SD).

Effects of vaccination on blood titer of Influenza-specific IgG were analysed by non-parametric and repeated measures Friedman test because the distribution of this parameter was not normal. This test was performed by comparing titer $\mathrm{d} 52$ versus titer $\mathrm{d} 95$

The effect of scFOS supplementation on blood Influenzaspecific IgG was analyzed as follow: difference between titer obtained the $\mathrm{d}$ before the first vaccination and titer obtained after the vaccination (at the age of 74 and $95 \mathrm{~d}$ ) was calculated. Four titer categories $(<20 ; 20 ; 40$ and $>40)$ were identified according to the distribution of the values and based on LDA practices. A Chi-square test evaluated if there was an association between diets and titer (i.e., association between diets and titer d 74 - titer d 52, first Chi-square test; and titer d 95 - titer d 52, second Chi-square test). When relevant, the contingency table was analyzed by a Factorial Correspondence Analysis ( $R$. Software, Library FactoMineR; [16] in order to illustrate these associations.

For all results, the significance of the results was set at $p<0.05$ while a trend was reported for $p<0.10$.

\section{Results}

No sanitary problems that could have disrupted the trial occurred. Both scFOS and control diets were well consumed by the sows and their piglets. Thus, we assume that experimental conditions were met to achieve the aims of the study.

\section{Characteristics of piglets at birth}

The diets did not alter the total number of born piglets $(15.2 \pm$ $2.89)$, the number of piglets born alive (14.4 \pm 1.79$)$, mean BW of the litter at birth $(18.6 \pm 1.05 \mathrm{~kg})$, and the mortality rate of the piglets within the first $48 \mathrm{~h}(15.6 \pm 3.05 \%)$. After standardization, litter size and average BW were similar among diets (Table 3).

Table 3: Characteristics of piglets from sows fed a control (CTRL) or a short-chain fructooligosaccharides (scFOS) supplemented diet.

\begin{tabular}{|c|c|c|c|c|}
\hline \multirow[b]{2}{*}{ Item } & \multicolumn{2}{|c|}{ Diet } & \multicolumn{2}{|c|}{$p$-value } \\
\hline & CTRL & scFOS & SD & scFOS \\
\hline Number of sows & 64 & 65 & & \\
\hline Average litter size ${ }^{1}$ at birth (born) & 14.9 & 15.4 & 2.89 & 0.362 \\
\hline
\end{tabular}




\begin{tabular}{|c|c|c|c|c|}
\hline Average litter size ${ }^{1}$ at birth (born alive) & 14.2 & 14.6 & 1.79 & 0.55 \\
\hline$\%$ of death between birth and $48 \mathrm{~h}$ & 15.8 & 15.3 & 3.05 & 0.803 \\
\hline Mean litter BW at birth, kg & 18.5 & 18.7 & 1.05 & 0.776 \\
\hline Average litter size after standardization & 12.4 & 12.7 & 2.04 & 0.154 \\
\hline Average BW of litter after standardization, $\mathrm{kg}$ & 16.3 & 16.4 & 0.667 & 0.799 \\
\hline
\end{tabular}

\section{Reproductive performance of sows}

Diet did not influence body temperature $24 \mathrm{~h}$ after farrowing, but dietary supplementation with scFOS reduced farrowing duration ( $p=0.012$, Table 4).

Diet did not modify feed intake of sows from d 109 of gestation to weaning nor back fat thickness at farrowing. Supplementation with scFOS tended to preserve back fat thickness at weaning in comparison to maltodextrins $(+0.8 \mathrm{~mm}$, $p=0.091$; Table 4).

Adding scFOS in the diet of the sows increased colostrum ash content $(+0.21 \%$; $p<0.05)$, decreased fat percentage $(-1.88 \%$; $\mathrm{p}=0.006)$, energy content $(-0.69 \mathrm{~kJ} / \mathrm{g} ; \mathrm{p}=0.009)$, and tended to decrease total carbohydrates $(-0.55 \% ; \mathrm{p}=0.061)$ and dry matter $(-1.6 \% ; p=0.095)$ (Table 5). It did not modify CP that averaged $15.6 \pm 1.53 \%$. Supplementation with scFOS did not modify levels of total IgA in colostrum that averaged $10.6 \pm 2.11 \mathrm{mg} / \mathrm{mL}$. An interaction between sow parity and scFOS supplementation was observed on IgG levels $(p=0.035)$. More precisely, scFOS increased IgG in oldest sows (parity 4 and higher; $p=0.007$; Figure 1) but not in youngest.

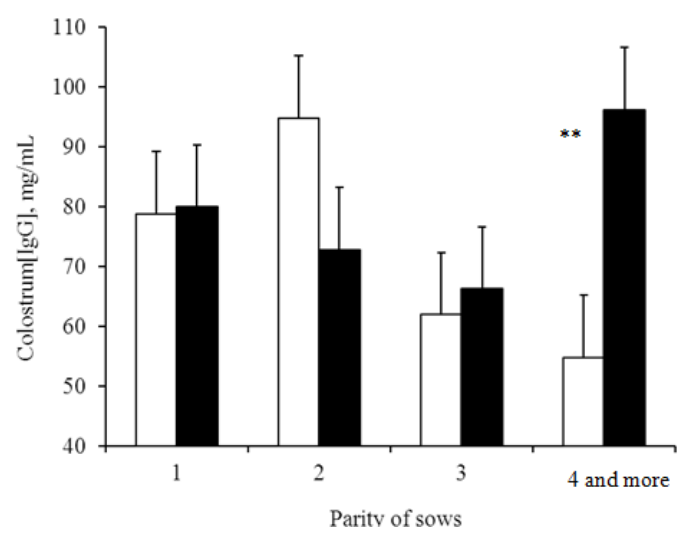

Figure 1: Effects of short-chain fructooligosaccharides (scFOS) supplementation and of sow parity on colostral IgG levels. ( $n=16$ sows in CTRL group and 17 sows in scFOS group). White bar: CTRL; Black bar: scFOS; ${ }^{* *}: \mathrm{P}<0.01$. Data as means \pm standard deviation of the model.

Table 4: Characteristics of sows fed a control (CTRL) or a short-chain fructooligosaccharides (scFOS) supplemented diet at farrowing and weaning.

\begin{tabular}{|l|l|l|l|l|}
\hline & Diet & \multicolumn{2}{l|}{ p-value } \\
\hline Item & CTRL & scFOS & SD & \\
\hline Number of sows & 64 & 65 & & \\
\hline Farrowing duration, $\mathrm{h}$. & 3.23 & 2.59 & 1.18 & 0.012 \\
\hline Temperature $24 \mathrm{~h}$ after farrowing, ${ }^{\circ} \mathrm{C}$ & 38.6 & 38.6 & 0.27 & 0.592 \\
\hline Feed Intake, $\mathrm{kg} / \mathrm{d}$ & 7.4 & 7.3 & 0.34 & 0.518 \\
\hline Back fat thickness at farrowing, $\mathrm{mm}$ & 17.9 & 18.2 & 2.58 & 0.357 \\
\hline Back fat thickness at weaning, $\mathrm{mm}$ & 14.1 & 14.9 & 4.4 & 0.091 \\
\hline
\end{tabular}

Table 5: Colostrum nutritional and immune quality in sows fed a control (CTRL) or a short-chain fructooligosaccharides (scFOS) supplemented diet.

\begin{tabular}{|l|l|l|l|}
\hline & Diet & P-value & SD \\
\hline Item & CTRL & scFOS & \\
\hline Number of sows & 16 & 17 & \\
\hline Nutritional composition & & \\
\hline
\end{tabular}




\begin{tabular}{|l|l|l|l|l|}
\hline $\mathrm{DM}, \%$ & 24.9 & 23.3 & 4.84 & 0.095 \\
\hline $\mathrm{CP}, \%$ & 15.3 & 15.8 & 1.53 & 0.588 \\
\hline Fat, \% & 6.39 & 4.51 & 1.78 & 0.006 \\
\hline Carbohydrates, \% & 2.73 & 2.18 & 1.57 & 0.061 \\
\hline Energy, kJ/g & 5.43 & 4.74 & 0.7 & 0.009 \\
\hline Ash, \% & 0.51 & 0.72 & 0.59 & 0.0001 \\
\hline Immune quality & 72.6 & 78.8 & 17.8 & 0.409 \\
\hline IgG, mg/mL & 10.1 & 10.9 & 2.11 & 0.664 \\
\hline IgA, mg/mL & \multicolumn{3}{|l|}{} \\
\hline
\end{tabular}

\section{Growth performance of suckling piglets}

Neither total number of weaned piglets nor piglet mortality rate (between $\mathrm{d} 2$ and weaning) was influenced by diet (11.3 \pm
0.06; Table 6). At the age of $20 \mathrm{~d}, \mathrm{BW}$ and Average Daily Gain (ADG) of piglets were similar whatever the diet of their dam (Table 6).

Table 6: Growth performance of suckling piglets of sows fed a control (CTRL) or a short-chain fructooligosaccharides (scFOS) supplemented diet.

\begin{tabular}{|c|c|c|c|c|}
\hline \multirow[b]{2}{*}{ Item } & \multicolumn{2}{|l|}{ Diet } & \multicolumn{2}{|c|}{ p-value } \\
\hline & CTRL1 & scFOS & SD & scFOS \\
\hline Number of sows & 64 & 65 & & \\
\hline Number of weaned-piglets & 11.3 & 11.3 & 0.06 & 0.965 \\
\hline$\%$ of deaths between $48 \mathrm{~h}$ and weaning & 7.08 & 5.48 & 8.99 & 0.193 \\
\hline $\mathrm{ADG}^{*}$ per piglet, $\mathrm{g}$ & 260 & 269 & 46.1 & 0.211 \\
\hline Piglet $\mathrm{BW}^{*}$ at weaning, $\mathrm{kg}$ & 6.16 & 6.29 & 0.66 & 0.365 \\
\hline
\end{tabular}

\section{Growth performance of pigs during the breeding period}

The average mortality rate was not significantly affected by dietary treatments (Table 7). Supplementation with scFOS of piglets during the pre-starter phase significantly increased ADG in all periods ( $p \leq 0.001$, Table 7 ), while it increased when piglets come from scFOS supplemented sows on starter phase $(p=0.048)$. Furthermore, a significant interaction was obtained between sow diet and piglet diet at the beginning of the growing phase ( $p=0.031$, Table 7$)$. ADI of piglets significantly decreased during starter phase when their dam received scFOS $(p=0.003)$, while it tended to decrease in pre-growing phase when piglets were supplemented with scFOS. Overall, FCR was improved during all the breeding period when piglets received scFOS in pre-starter phase and in the starter phase when their dam were supplemented (Table 7). BW was higher in pregrowing and growing phases when piglets received scFOS during the pre-starter phase (Table 7).

Table 7: Effects of early-life short-chain fructooligosaccharides (scFOS) supplementation on growth performance of pigs from starter to end of growing phase.

\begin{tabular}{|c|c|c|c|c|c|c|c|c|}
\hline \multicolumn{4}{|c|}{ Diets } & \multicolumn{5}{|c|}{ P-value } \\
\hline & $\begin{array}{l}\text { CTRL/ } \\
\text { CTRL }\end{array}$ & $\begin{array}{l}\text { CTRL/ } \\
\text { scFOS }\end{array}$ & $\begin{array}{l}\text { scFOS/ } \\
\text { CTRL }\end{array}$ & $\begin{array}{l}\text { scFOS/ } \\
\text { scFOS }\end{array}$ & SD & Sow & Piglet & Sow ${ }^{*}$ piglet \\
\hline \multicolumn{9}{|c|}{ Body Weight, kg } \\
\hline 40 days $^{2}$ & 10.9 & 11.3 & 10.9 & 11.1 & 1.38 & 0.878 & 0.575 & 0.861 \\
\hline 64 days $^{2}$ & 23.6 & 24.9 & 23.6 & 24.2 & 1.68 & 0.599 & 0.173 & 0.608 \\
\hline
\end{tabular}




\begin{tabular}{|c|c|c|c|c|c|c|c|c|}
\hline 103 days $^{2}$ & $56.4^{\mathrm{a}}$ & $58.4^{\mathrm{b}}$ & $56.3^{a}$ & $58.1^{b}$ & 1.92 & 0.815 & 0.024 & 0.866 \\
\hline 148 days $^{2}$ & $95.6^{\mathrm{a}}$ & $98.8^{\mathrm{b}}$ & $96.7^{\mathrm{a}}$ & $99.6^{b}$ & 3.75 & 0.383 & 0.006 & 0.925 \\
\hline \multicolumn{9}{|c|}{ Average Daily Intake, g } \\
\hline 40-64 days & $935^{\mathrm{ab}}$ & $963^{b}$ & $909^{a}$ & $902^{\mathrm{a}}$ & 30 & 0.003 & 0.406 & 0.182 \\
\hline 64-103days & 1795 & 1781 & 1784 & 1737 & 42.2 & 0.121 & 0.091 & 0.369 \\
\hline $\begin{array}{l}103-148 \\
\text { days }\end{array}$ & 2543 & 2557 & 2552 & 2551 & 38.5 & 0.929 & 0.771 & 0.361 \\
\hline \multicolumn{9}{|c|}{ Average Daily Gain, g } \\
\hline 40-64 days & $528.4^{a}$ & $567^{b}$ & $527.5^{\mathrm{a}}$ & $543.2^{\mathrm{a}}$ & 14.2 & 0.048 & $<0.001$ & 0.062 \\
\hline 64-103 days & $840.4^{a}$ & $858.6^{\mathrm{b}}$ & $839.3^{\mathrm{a}}$ & $869.9^{b}$ & 14.3 & 0.406 & 0.001 & 0.309 \\
\hline $\begin{array}{l}103-148 \\
\text { days }\end{array}$ & $883.5^{\mathrm{a}}$ & $947.7^{\mathrm{b}}$ & $907.3^{c}$ & $935.2^{\mathrm{b}}$ & 24.6 & 0.216 & $<0.001$ & 0.031 \\
\hline \multicolumn{9}{|c|}{ Feed Conversion Ratio } \\
\hline 40-64 days & $1.77^{\mathrm{a}}$ & $1.7 \mathrm{~b}^{\mathrm{c}}$ & $1.72^{\mathrm{b}}$ & $1.66^{\mathrm{c}}$ & 0.032 & 0.004 & $<0.001$ & 0.542 \\
\hline $64-103$ days & $2.14^{a}$ & $2.07^{\mathrm{b}}$ & $2.12^{\mathrm{a}}$ & $2.03^{\mathrm{b}}$ & 0.042 & 0.15 & $<0.001$ & 0.484 \\
\hline $\begin{array}{l}103-148 \\
\text { days }\end{array}$ & $2.88^{\mathrm{a}}$ & $2.72^{\mathrm{b}}$ & $2.82^{\mathrm{c}}$ & $2.72^{\mathrm{b}}$ & 0.073 & 0.182 & $<0.001$ & 0.086 \\
\hline \multicolumn{9}{|l|}{ Mortality, \% } \\
\hline 40-64 days & 0.59 & 1.64 & 1.12 & 1.12 & 2 & 0.988 & 0.535 & 0.535 \\
\hline $64-103$ days & 1.51 & 0.96 & 0.95 & 0.98 & 2.08 & 0.748 & 0.762 & 0.734 \\
\hline $\begin{array}{l}103-148 \\
\text { days }\end{array}$ & 0.6 & 0.43 & 0.63 & 1.81 & 2.18 & 0.428 & 0.13 & 0.525 \\
\hline
\end{tabular}

\section{Growth performance of pigs at slaughtering}

Data recorded at slaughtering came from 769 pigs: 191, 194, 193, and 191 pigs for the CTRL/CTRL; CTRL/SCFOS; SCFOS/CTRL; and scFOS/scFOS groups respectively (Figure 2 ).

Cold carcass weight was similar whatever the treatments (Figure 2). At slaughtering, the piglets more rapidly reached the target body weight of $120 \mathrm{~kg}$ when they received scFOS diet (185.2 and $184.3 \mathrm{~d}$ for CTRL/scFOS and scFOS/scFOS groups) or when their dam received it $(186.9$ for SCFOS/CTRL group; $\mathrm{p}=0.0001$, Figure 2 ).

The shortest growing period was obtained with the scFOS/ scFOS group and the longest with the CTRL/CTRL group (184.3 d versus $192.9 \mathrm{~d}$ ). Lean meat content was higher when pigs were supplemented with scFOS during the pre-starter phase while it tended to be lower when sows were supplemented with scFOS $(p=0.06$, Figure 2$)$.

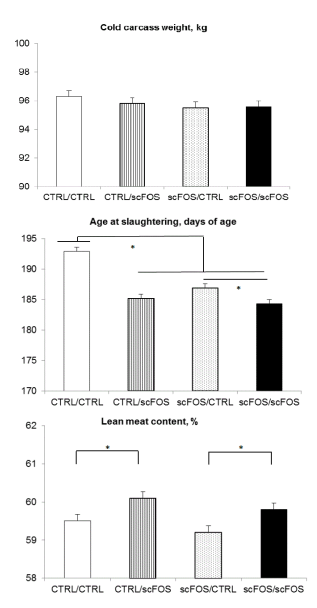

Figure 2: Effect of an early-life supplementation with scFOS on growth performance at slaughtering. Individual data. Data are adjusted means \pm SD. White bar: CTRL/CTRL; Hatched bar: CTRL/scFOS; Dotted bar: scFOS/CTRL; Black bar: scFOS/scFOS. 


\section{Blood levels of Influenza-specific IgG}

Time before or after the vaccination significantly affected titers of specific blood anti-Influenza IgG $(p<0.05)$, meaning that the vaccination was efficient to create an immune response whatever the diets.

Furthermore, blood titer levels differences were significantly associated with scFOS supplementation at d 95 (association between diets and titer d 95 - titer d 52; Chi-square=15.54; $p=0.05)$ but not at $d 74(p>0.05)$.

Correspondence analysis (Figures 3 and 4) showed that scFOS/scFOS group was associated with titer category $>40$ and 20, while CTRL/scFOS group was associated with the titer category 40. CTRL/CTRL and SCFOS/CTRL groups were associated with titer categories $<20$ and 20.

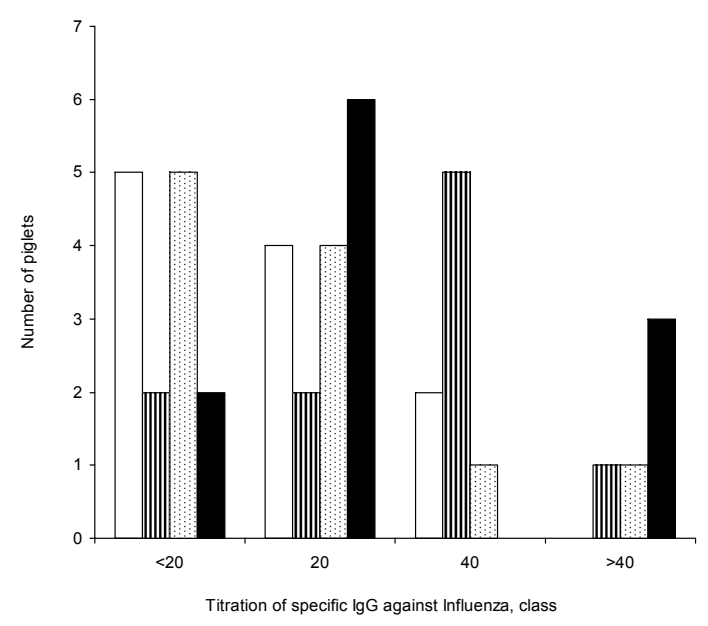

Figure 3: Number of piglets per Influenza IgG titer categories according to short-chain fructooligosaccharides (scFOS) supplementation of sows and piglets. Data used were delta of titer per d 95-titer d 52. CTRL: Control diet.

White bar: CTRL/CTRL; Hatched bar: CTRL/scFOS; Dotted bar: scFOS/CTRL; Black bar: scFOS/scFOS

\section{Discussion}

The importance of early-life nutrition on later performance and health is emerging. In this context, our study aimed at determining if a short-term supplementation of scFOS at the beginning of the life of the piglets may impact their immune response and their growth performance on later life. Additionally, reproductive performance of sows were assessed. The originality of our study compared to the previous one published on scFOS was to quantify in which manner a shortterm scFOS supplementation during early-life of the piglets impact their performance all along their life.

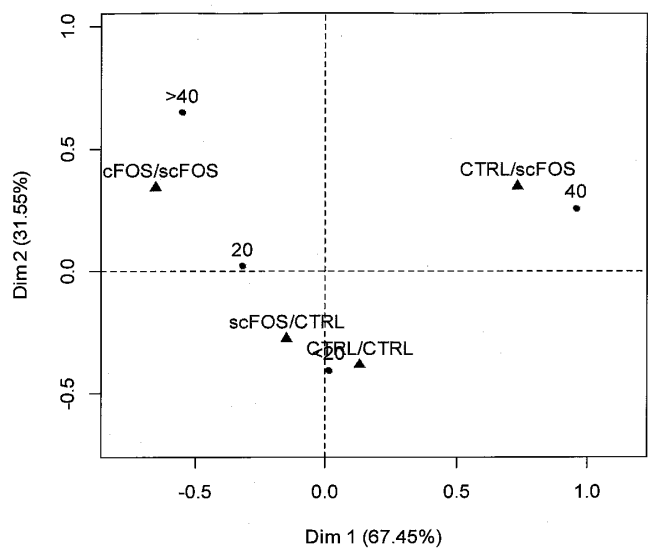

Figure 4: Association between diets of sows and piglets and titer of Influenza IgG (association related to diets, $p=0.05$ ). Data are obtained by Factorial Component Analysis. Data used were delta of titer per d 95-titer d 52. scFOS: ShortChain Fructooligosaccharides; CTRL: Control. Dim: Dimension. First axis represents $67.45 \%$ of inertia; second axis represents $31.55 \%$ of inertia.

\section{Supplementation of sows with scFOS modifies reproductive performance}

Five ds of scFOS supplementation reduced farrowing duration. That is of great importance for piglets' survival and for subsequent reproductive performance of sows [17]. Many factors can influence farrowing duration, including breed, sow age, gestation length, litter size, sow body condition and constipation state [18]. Breed, sow age, gestation length, and litter size were similar in both sow groups. No measure of constipation state has been realised in the present study, but scFOS are known to regulate intestinal transit in humans [19]. Back fat thickness at weaning tended to be higher in sows fed scFOS compared to those fed control diet while feed intake was similar between the 2 groups. Le Bourgot et al. [11] observed similar effects on backfat thickness in sows fed with scFOS diet for the 4 last week of gestation and lactation. Late-pregnancy and lactation are specific physiological periods during which sows may become insulin-resistant and mobilize their energy storage $[4,20]$. As a prebiotic ingredient, scFOS modulate intestinal microbiota and increase VFA production in colon of many species, including pigs [21]. They also enhance insulin sensitivity in both healthy and obese animals [8,22]. The relationship between dietary scFOS supplementation, intestinal microbiota modulation and reduction of blood insulinemia has been recently highlighted in humanized gnotobiotic diet-induced obesity mice [23]. Thus, scFOS may have enhanced insulin sensitivity of sows and by this way reduced mobilization of adipose tissue for fatty acid synthesis during lactation.

All values reported for composition of colostrum, including fat content and energy are in agreement with biological values previously reported by Klobasa et al. [24] and Le Dividich et al. [13]. However, energy and fat contents were slightly lower in colostrum of sows receiving SCFOS than CTRL diet, while feed 
intake and number of suckling piglets were equivalent. This is consistent with a possible lower utilization of fat storage in sows fed scFOS mentioned above. It may also be related to the fact that, even if the procedure given by the laboratory in charge of the dosage was respected, the subsampling rate was not sufficient for an appropriate quality assessment of colostrum. This difference in nutritional composition did not impact the mortality rate of piglets or BW at weaning; suggesting piglets from sows fed the scFOS diet received sufficient energy quantity to ensure survival and growth.

\section{ScFOS supplementation results in long-term effects on growth performance}

Positive carry-over effects are observed on ADG and FCR during the breeding period when piglets or their dam received scFOS. Consequently, the animals reached the target body weight for slaughter at a younger age when sows and piglets were supplemented with scFOS. Similarly, Le Bourgot (INRA, Rennes, France, personal communication) observed a positive carry-over effect of scFOS supplementation of sows on BW of their piglets at the age of $77 \mathrm{~d}$. The scFOS also improved growth and feed efficiency when directly fed to veal calves [25] or piglets $[6,7]$. However, these previous studies evaluated the effects of scFOS on growth performance during supplementation, while the present study highlighted a positive carry-over effect of early-life nutrition on later growth performance and carcass characteristics. Numerous studies have been designed to evaluate impacts of early nutrition on piglet growth performance but they mainly focused on protein levels or on feed restriction [26,27]. To our knowledge, no data is available on the effects of prebiotics given to piglets on their later growth performance.

The modification of lean meat content at slaughtering suggest that scFOS may affect energy metabolism pathways when directly fed to piglets or to their lactating mothers, probably through a modification of milk composition in this case [10] Long-term effects of scFOS supplementation of sows and piglets are also undoubtedly related to a modulation of intestinal microbiota. Evidence for the involvement of underlying epigenetic modifications in long-term studies emerges with regard to the gastrointestinal tract. More precisely, microbiota is involved in modulating the host epigenome at the gut level $[28,29]$. Such results, in addition to their well-known prebiotic properties, lead to the idea that scFOS may modulate early bacterial colonization of piglets and by this way modulate metabolic responses later on.

\section{Effects of supplementation of sows with scFOS on immune quality of colostrum}

In the present study, supplementation of sows with scFOS did not modify colostral IgA levels. These results are inconsistent with the previous results reported in sows [10] and bitches [9]. The lack of effect may be related to the relatively short duration of scFOS supplementation at the end of gestation in the present study (i.e., 1 versus 4 week in others). All cell types involved in the immune response are present in the mammary gland early during gestation while a strong increase in lymphocyte count has been noticed between 80 and $90 \mathrm{~d}$ of gestation [30]. A large part of these cells originate from Gut-Associated Lymphoid Tissues (GALT) as they migrate by homing into the mammary gland. ScFOS supplementation is supposed to modify total IgA levels in colostrum by increasing immune cells from GALT and by favoring entero-mammary cell migration [10]. Thus, it can be supposed that supplementation for the last $5 \mathrm{~d}$ before farrowing is too short to stimulate lymphocyte migration from GALT to mammary gland.

Supplementation with scFOS did not alter colostrum IgG levels, except for the sows with higher parity ranking for which IgG levels were increased. There are inconsistent results regarding the influence of parity on IgG levels in colostrum [31,32]. A modification of colostral IgG levels could be related to a modulation of blood IgG levels: contrary to the major part of colostrum IgA, those coming from immune cell by homing, colostrum IgG are mainly provided by blood to the udder through passive diffusion [33].

\section{Supplementation of piglets with scFOS modulates immune response}

Influenza-vaccine used as model of immune challenge increased serum levels of Influenza-specific IgG, suggesting vaccination was efficient. Both mucosal and systemic immunities contribute to resistance against Influenza infection and disease. Secretory $\lg A(\operatorname{sg} A)$ are involved in the protection of the upper respiratory tract while serum IgG is involved in the protection of the lower respiratory tract [34]. The serum antibody response is dominated by Influenza IgG when vaccination is performed by intramuscular injection [34]. Thus, the measure of Influenza IgG in blood is supposed to well reflect the immune response of piglets after vaccination.

Supplementation of piglet diet with scFOS modulated immune response of piglets towards Influenza vaccination. Similarly, Le Bourgot et al. [11] observed a modulation of faecal and blood concentrations of specific $\operatorname{IgA}$ in piglets vaccinated against Influenza and supplemented with scFOS. They also observed a positive and significant relationship between blood specific IgA and IgG. Puppies intranasally vaccinated against Bordetella bronchiseptica showed a trend to higher response of specific IgM when diet with $1 \%$ scFOS was fed to bitches and puppies [9]. Vos et al. [35] showed a prebiotic mixture containing galacto- and fructo-oligosaccharides enhanced systemic adaptive immune responses in a murine model of Influenza vaccination.

In the current study, a relationship between piglet BW and stimulated immune response may be hypothesized. Indeed, piglets that received scFOS had a greater BW at $d 103$ of age compared to other groups. A faster growth of these piglets might result in a faster development of immune system and, in turn, in a boosted immune response after vaccination. However, such hypothesis does not work for sow supplementation. The effect of the sow supplementation with scFOS remains unclear. Indeed, piglets from sows fed with scFOS and receiving themselves scFOS grew as well as CTRL/scFOS group. CTRL/ scFOS group was associated with titer 40 while scFOS/scFOS 
group was associated with titer either $>40$ or 20 . The supplementation of the sows seems to be either additional to that of piglets or to negatively interact with. Le Bourgot et al. [10] recently demonstrated that the supplementation of the sow stimulated development of gut associated lymphoid tissues of suckling piglets. Perhaps the effect of sow supplementation is local, via early microbiota modulation, while direct intake of scFOS allows more systemic effects. To support this assumption, Le Bourgot et al. (oral communication accepted in Beneficial Microbes congress, October 2016) reported that the supplementation of sows with scFOS allows stimulating specific immune response against Lawsonia intracellularis, pathogen bacteria with intestinal tropism.

Overall, underlying mechanisms by which scFOS modulate immune system remain unclear but may be related to a modulation of intestinal microbiota. Vos et al. [35] showed enhancement of systemic adaptive immune responses in a murine model of Influenza vaccination was accompanied with increased proportions of certain members of intestinal microbiota. The key role of intestinal Gram-positive bacteria in controlling adaptive immunity after respiratory infection with influenza virus has been recently highlighted in mice [36], demonstrating that all commensal bacteria did not contribute equally to the immunocompetence in the respiratory tract. In pigs, Lactobacillus genus exhibits a significant decline at weaning [37]. A direct intake of scFOS that is widely known to increase lactobacilli in vivo [38,39] may prevent this post-weaning decrease and subsequently improve the efficiency of the immune response to vaccination. Growing body of evidences shows there are strong interrelationships between microbiota and both local and systemic immune systems of mammalians [40].

\section{Conclusion}

These data suggest that scFOS-supplementation of piglets during early-life results in gains for breeders directly by sustaining good growth performance of pigs and indirectly by preserving the reproductive herd.

Furthermore, these data suggest there are sustainable immune, metabolic and zootechnical modifications due to earlylife supplementation with scFOS. Indeed, supplementation of newborn piglets with scFOS improved zootechnical performance at slaughter and modulated response to an immune challenge such as vaccination. This supplementation also modified the fat percentage on carcass, suggesting changes in energy metabolism pathways. The mechanisms underlying these effects of feeding scFOS to sows and piglets need to be further investigated but are most likely related to the composition and activities of intestinal microbiota.

\section{Funding}

This study received funding from Beghin-Meiji, a company producing short-chain fructooligosaccharides belonging to the company "Tereos". FR and EA are employed by Tereos. This study received funding from Jefo Europe, a company which sells short-chain fructooligosaccharides.

\section{Acknowledgement}

The authors gratefully thank the employees of LDA 22 for their help with laboratory analysis and the breeders of pigs for their active participation. They also gratefully thank Jean-Yves Dourmad from INRA of Rennes for reading the publication and for his advices. They sincerely thank Samuel Voller from University of Plymouth for editing the manuscript for English style.

\section{Author's Contribution}

EA, CM conceived, designed and performed the experiments described in this work and drafted the manuscript. CM, EA, FR participated in the design of the study and statistical analysis. $E A, C M, F R, A W, J C B$ participated in the design of the study, interpretation of data and critical revision of the manuscript. All authors read and approved the final manuscript.

\section{References}

Guilloteau P, Zabielski R, Hammon HM, Metges CC (2010) Nutritional programming of gastrointestinal tract development. Is the pig a good model for man? Nutr Res Rev 23: 4-22.

2. Lallès JP (2012) Long term effects of pre- and early postnatal nutrition and environment on the gut. Journal of Animal Sciences 90: 421-429.

3. Boulot S, Quesnel H, Quiniou N (2008) Management of high prolificacy in French herds: Can we alleviate side effects on piglet survival. Advances in Pork Production 19: 213-220.

4. Père $M C$, Etienne $M$ (2007) Insulin sensitivity during pregnancy, lactation and post weaning in primiparous gilts. JAS 85: 101-110.

5. Gibson GR, Probert H, van Loo J, Rastall RA, Roberfroid MB (2004) Dietary modulation of the human colonic microbiota: Updating the concept of prebiotics. Nutrition Research Review 17: 259-275.

6. Howard MD, Liu H, Spencer JD, Kerley MS, Allee GL (1999) Incorporation of short-chain fructooligosaccharides and tylan into diets of early-weaned pigs. UMC Animal Sciences Departmental Report, University of Missouri. pp: 90-95.

Xu C, Chen X, Ji C, Ma Q, Hao K (2005) Study of the application of fructo-oligosaccharides in piglets. Asian Australas. J Anim Sci 18: 1011-1016.

8. Respondek F, Swanson KS, Belsito KR, Vester BM, Wagner A, et al. (2008) Short-chain fructooligosaccharides influence insulin sensitivity and gene expression of fat tissue in obese dogs. J Nutr 138: $1712-1718$

9. Adogony V, Respondek F, Biourge V, Rudeaux F, Delaval J, et al. (2007) Effects of dietary scFOS on immunoglobulins in colostrum and milk of bitches. J Anim Physiol Anim Nutr 91: 169-174.

10. Le Bourgot C, Ferret-Bernard S, Le Normand L, Savary G, Menendez-Aparicio E, et al. (2014) Maternal short-chain fructooligosaccharide supplementation influences intestinal immune system maturation in piglets. PLos ONE 9: e107508.

11. Le Bourgot C, Ferret-Bernard S, Blat S, Apper E, Le Huerou-Luron I (2016) scFOS supplementation during gestation and lactation or after weaning differentially impacts pig growth and IgA response to Influenza vaccination. Journal of Functional Foods 24: 307-315. 
12. Le Dividich J, Martineau G, Thomas F, Demay H, Renoult $H$, et al. (2004) Acquisition de l'immunité passive chez les porcelets et production de colostrum chez la truie. Journées de la Recherche Porcine en France 36: 451-455.

13. Palmer DF, Coleman MT, Dowdle WR, Schild DC (1975) Advanced laboratory techniques for influenza diagnosis. In Immunology Series No. 6, Procedural Guide. Washington, DC: US Department of Health, Education and Welfare. pp: 65-75.

14. SAS Intitute. SAS User's Guide: Statistics (1990) Version 6, Edition 4,SAS Inst. Inc., Cary, NC.

15. Gill JL (1978) Completely randomized designs of analysis of variance. In Design and Analysis of Experiments in the Animal and Medical Sciences; lowa State University Press, Ames, USA 1: 135-258.

16. Cornillon PA, Guyader A, Husson F, Jégou N, Josse J, et al. (2008) Statistiques avec R. Presses Universitaires de Rennes, Rennes, France.

17. Friend DW, Cunningham HM, Nicholson JWG (1962) The duration of farrowing in relation to the reproductive performance of Yorkshire sows. Can J Comp Med Vet Sci 26: 127-131.

18. Oliviero, C, Heinonen M, Valros A, Peltoniemi O (2010) Environmental and sow-related factors affecting the duration of farrowing. Anim Reprod Sci 119: 85-91.

19. Paineau D, Payen F, Panserieu S, Coulombier G, Sobaszek A, et al. (2008) The effects of regular consumption of short-chain fructooligosaccharides on digestive comfort of subjects with minor functional bowel disorders. British Journal of Nutrition 99: 311-318.

20. Farmer C, Quesnel H (2009) Nutritional, hormonal, and environmental effects on colostrum in sows. JAS 87: 56-64.

21. Tsukahara T, Iwasaki Y, Nakayama K, Ushida K (2003) Stimulation of butyrate production in the large intestine of weaning piglets by dietary fructooligosaccharides and its influence on the histological variables of the large intestinal mucosa. Journal of Nutraceutical Science and Vitaminology 49: 414-421.

22. Massimino SP, McBurney MI, Field CJ, Thomson ABR, Hayek M, et al. (1998) Fermentable dietary fiber increases GLP-1 secretion and improves glucose homeostasis despite increased intestinal glucose transport capacity in healthy dogs. J Nutr 128: 1786-1793.

23. Respondek F, Gerard P, Bossis M, Boschat L, Bruneau A, et al. (2013) Short-chain fructo-oligosaccharides modulate intestinal microbiota and metabolic parameters of humanized gnotobiotic diet induced obesity mice. PLOS ONE 8: e71026.

24. Klobasa F, Werhahn E, Butler JE (1987) Composition of sow milk during lactation. JAS 64: 1458-1466.

25. Grand E, Respondek F, Martineau C, Detilleux J, Bertrand G (2013) Effects of short-chain fructooligosaccharides on growth performance of preruminant veal calves J Dairy Sci 96: 1094-1101.

26. Sarr O, Louveau I, Kalbe C, Metges C, Rehfeldt C, et al. (2010) Prenatal exposure to maternal low or high protein diets induces modest changes in the adipose tissue proteome of newborn piglets. JAS 88: 1626-1641.

27. Sarr O, Gondret F, Jamin A, Le Huërou-Luron I, Louveau I (2011) A high-protein neonatal formula induces a temporary reduction of adiposity and changes later adipocyte physiology. American Journal of Physiology, Regul, Integrative and Comparative Physiology 300: R387-R397.

28. Hinnebusch BF, Henderson JW, Siddique A, Malo MS, Zhang W, et al. (2003) Transcriptional activation of the enterocyte differentiation marker intestinal alkaline phosphatase is associated with changes in the acetylation state of histone $\mathrm{H} 3$ at a specific site within its promoter region in vitro. J Gastrointest Surg 7: 237-244.

29. Takahashi YW, Sugi Y, Nakano K, Tsuda M, Kurihara K (2011) Epigenetic control of the host gene by commensal bacteria in large intestinal epithelial cells. Journal of Biology and Chemistry 286: 35755-35762.

30. Salmon H, Delouis C (1982) Cinétique des sous-populations lymphocytaires et des plasmocytes dans la mamelle de truie primipare en relation avec la gestation et la lactation. Annales de la Recherche Veterinaire 3: 41-49.

31. Inoue T, Kitano K, Inoue K (1980) Possible factors influencing the immunoglobulin $\mathrm{G}$ concentration in swine colostrum. American Journal of Veterinary Research 41: 1134-1136.

32. Bland IM, Rooke JA (1988) Effects on colostrum immunoglobulin $G$ (IgG) concentrations and piglet colostrum intake of sow, udder section and time. British Society of Animal Sciences. pp: 158-162.

33. Salmon H (2011) Transmission de l'immunité maternelle chez le porc et les ruminants après la naissance. Bulletin Acta Veterinaires France 164: 203-210.

34. Cox RJ, Brokstad KA, Ogra PL (2004) Influenza virus: immunity and vaccination strategies. Comparison of the immune response to inactivated and live, attenuated influenza vaccines. Scand J Immunol 59: 1-15.

35. Vos AP, Haarman M, Buco A, Govers M, Knol J, et al. (2006) A specific prebiotic oligosaccharide mixture stimulates delayed-type hypersensitivity in a murine influenza vaccination model. International Immunopharmacology 6: 1277-1286.

36. Ichinohe T, Pang IK, Kumamoto Y, Peaper DR, Ho JH, et al. (2011) Microbiota regulates immune defense against respiratory tract influenza A virus infection. Proc Natl Acad Sci U S A 108: 5354-5359.

37. Mach N, Berri M, Estelle J, Levenez F, Lemonnier G, et al. (2015) Early-life establishment of the swine gut microbiome and impact on host phenotypes. Environmental Microbiology Reports 7: 554-569.

38. Pan XD, Chen FQ, Wu TX, Tang HG, Zhao ZY (2009) Prebiotic oligosaccharides change the concentrations of short-chain fatty acids and the microbial population of mouse bowel. J Zhejiang Univ Sci B 10: 258-263.

39. Le Blay G, Michel C, Blottiere HM, Cherbut C (1999) Prolonged intake of fructo-oligosaccharides induces a short-term elevation of lactic acid-producing bacteria and a persistent increase in cecal butyrate in rats. J Nutr 129: 2231-2235.

40. Maynard CL, Elson CO, Hatton RD, Weaver CT (2012) Reciprocal interactions of the intestinal microbiota and immune system. Nature 489: 231-241. 